\title{
The center-to-limb behavior of solar active regions at ultraviolet wavelengths
}

\author{
P. C. Crane ${ }^{1}$, L. E. Floyd ${ }^{2}$, J. W. Cook ${ }^{3}$, L. C. Herring ${ }^{2}$, E. H. Avrett ${ }^{4}$, and D. K. Prinz ${ }^{3, \star}$ \\ 1 Code 7213, Remote Sensing Division, Naval Research Laboratory, Washington, DC 20375, USA \\ 2 Interferometrics Inc., 14120 Parke Long Court, Suite 103, Chantilly, VA 20151, USA \\ e-mail: linton.floyd@nrl.navy.mil; herring@susim.nrl.navy.mil \\ 3 Code 7668, E.O. Hulburt Center for Space Research, Naval Research Laboratory, Washington, DC 20375, USA \\ e-mail: john.cook@nrl.navy.mil \\ ${ }^{4}$ Harvard-Smithsonian Center for Astrophysics, 60 Garden Street, Cambridge, MA 02138, USA \\ e-mail: eavrett@cfa.harvard.edu
}

Received 9 April 2003 / Accepted 14 January 2004

\begin{abstract}
The time series of solar ultraviolet irradiances measured by the Solar Ultraviolet Spectral Irradiance Monitor on the Upper Atmosphere Research Satellite have been analyzed to describe the center-to-limb behavior of the excess surface brightness of solar active regions over the wavelength range 142-265 $\mathrm{nm}$. Comparison of these results with the analysis by Worden, Woods, and Bowman (2001, ApJ, 560, 1020) of the time series produced by the Solar-Stellar Irradiance Comparison Experiment on the same spacecraft over the wavelength range 120-170 nm shows excellent agreement. For 170-265 nm, we present new results on the center-to-limb behavior of the surface brightness of solar active regions. Comparisons with previous results for the quiet disk show roughly similar behaviors at wavelengths below $168 \mathrm{~nm}$ and above $210 \mathrm{~nm}$, where both exhibit weak limb brightening and darkening, respectively. At intermediate wavelengths, $168-210 \mathrm{~nm}$, active regions exhibit much stronger limb darkening than does the quiet disk. Our Fourier analysis and the multi-component modeling of Worden et al. $(2001, \mathrm{ApJ}, 560,1020)$ are found to be complementary and could be productively combined in future work. We also compare our results with a similar analysis based upon the semi-empirical model atmospheres of Fontenla et al. (1999, ApJ, $518,480)$, further improved by Avrett. We compare the measurement- and model-based analyses and suggest the direction of improvements needed in the model atmospheres.
\end{abstract}

Key words. Sun: activity - Sun: faculae, plages - Sun: UV radiation - Sun: rotation - Sun: chromosphere - Sun: photosphere

\section{Introduction}

The large rotational modulation of solar irradiances at ultraviolet wavelengths (Lean 1987; Lean et al. 1992; Floyd et al. $1998 \mathrm{~b})$ is the consequence of the enhanced surface brightness of solar active region plage compared to the quiet disk of the Sun. The degree of that enhancement is the solar contrast and often is defined in terms of the excess surface brightness (Chapman \& McGuire 1977):

$C_{\lambda}(\mu)=I_{\lambda}^{\mathrm{e}}(\mu) / I_{\lambda}^{\mathrm{d}}(\mu)$,

where $\mu$ is the cosine of the heliocentric angle, $\theta ; I_{\lambda}^{\mathrm{d}}(\mu)$, the surface brightness of the quiet disk; and $I_{\lambda}^{\mathrm{e}}(\mu)$, the excess surface brightness of an active region,

$I_{\lambda}^{\mathrm{e}}(\mu)=I_{\lambda}^{a}(\mu)-I_{\lambda}^{\mathrm{d}}(\mu)$.

The "excess" contrast is used here because the rotational modulation is proportional to the excess surface brightness (the more

Send offprint requests to: $\mathrm{P}$. Crane,

e-mail: patrick.crane@nrl.navy.mil

^ Dr. Dianne K. Prinz (1938-2002). common definition is given as $\left.C_{\lambda}(\mu)+1\right)$. Knowledge of the behavior of the contrast as a function of wavelength is essential to ongoing efforts to model the variations of solar ultraviolet irradiances, whether from spatially resolved observations of the Ca II K chromosphere (Lean et al. 1982; Lean \& Skumanich 1983; Lean 1984, 1988) or from solar magnetograms (Fligge et al. 2000). At ultraviolet wavelengths, unfortunately, actual measurements of the contrast are rare (Hersé 1979; Cook et al. 1980; Morrill et al. 2001) and most of our knowledge is either indirect (Lean 1984) or theoretical (Fontenla et al. 1999).

Because of the radial temperature and density structure of the solar atmosphere, the surface brightnesses of both active regions and the quiet disk are expected to vary with heliocentric angle from the center of the solar disk (Fontenla et al. 1999), and unless the dependencies with angle are the same, the solar contrast also will exhibit center-to-limb variations. "Limb brightening" and "limb darkening" occur, respectively, when the surface brightness increases or decreases as the limb is approached. The center-to-limb behavior of the contrast reflects the competition between the numerator and denominator in the ratio of Eq. (1). The behavior of the 
quiet disk at ultraviolet wavelengths is well known from direct measurement (Bonnet 1968; Kjeldseth Moe \& Milone 1978; Basri et al. 1979; Samain 1979, 1980; Cook \& Kjeldseth Moe 1982; Roussel-Dupré 1982; Brekke \& Kjeldseth-Moe 1994a,b; Greve \& Neckel 1996; Hestroffer \& Magnan 1998; Wilhelm et al. 1998; Dammasch et al. 1999). Consequently, because of Eq. (1), knowledge of the center-to-limb behaviors of active regions and of their contrasts are equivalent. Yet little is known about either because of the difficulty of directly measuring the intensities of individual active regions, even at optical wavelengths (Wang \& Zirin 1987; Unruh et al. 1999). At ultraviolet wavelengths, only qualitative results, with limited wavelength coverage and/or spectral resolution, have been available (Prinz 1974; Hersé 1979). Consequently, modelers have assumed the contrast is constant (Cook et al. 1980; Lean 1984) or used theoretical values (Fontenla et al. 1999).

Lacking direct quantitative measurements of spatially resolved active regions at ultraviolet wavelengths, the only available and relevant observations are long-term time series of solar ultraviolet irradiances measured by space-based ultraviolet spectrometers. Two such experiments continue to operate onboard the Upper Atmosphere Research Satellite (UARS) - the Solar Ultraviolet Spectral Irradiance Monitor (SUSIM; Brueckner et al. 1993) and the Solar-Stellar Irradiance Comparison Experiment (SOLSTICE; Rottman et al. 1993; Woods et al. 1993). In a recent study, Worden et al. (2001, hereafter WWB) have analyzed the SOLSTICE time series by fitting multiple components in the time domain. Unfortunately, their results are limited by systematic uncertainties to the wavelength range $120-170 \mathrm{~nm}$. The limitations of the SUSIM are different and, as we will see, the useful wavelength coverage extends and complements that of the SOLSTICE.

We analyze the SUSIM time series in the frequency domain, extending the Fourier analyses of previous investigators, in particular, the work of Donnelly and his collaborators $(1982,1986,1990)$. Their analyses of the solar UV irradiance data from the Solar Backscatter UltraViolet (SBUV) experiment aboard Nimbus-7, the UV spectrometer onboard the Solar Mesosphere Explorer, and the Ly- $\alpha$ channel of the Atmosphere Explorer $E$ found that the ratios of 13-day power to 27-day power at far- and mid-uv wavelengths (100-200 nm and 200-300 nm) are significant for different time intervals. Both the center-to-limb behavior of active regions, i.e., limb brightening and darkening (Ward \& Shapiro 1961, 1962; Shapiro $1965 \mathrm{a}, \mathrm{b})$, and the tendency for active regions to occur separated by $\sim 180^{\circ}$ in longitude, i.e., two-sidedness (Kiepenheuer 1953), are important factors in determining the values of those ratios. Stronger limb darkening and stronger two-sidedness both increase the value of the ratio.

The values of the ratios vary with time because the tendency for two-sidedness interacts with other factors including the longitudinal spread and evolution of active regions. Potentially, if that variability could be somehow "calibrated", as is done in other areas of astronomy that use Fourier analysis - radio, optical, and speckle interferometry - the two factors could be separated. In this analysis, our knowledge of the center-to-limb behaviors of the international sunspot number, $R$, and the solar radio flux density, $F_{10.7 \mathrm{~cm}}$, will provide the means to do so. Because limb darkening is weak for both indices, an episode of strong two-sidedness is optimal for this study. Such an episode of unusual $\sim 13.5$-day variations occurred during the UARS mission from 1994 September 27 to 1995 June 4 (Floyd et al. 1997; Crane 1998). DeLand \& Cebula (1998) also report $\sim 13.5$-day variations for the same time period in the 205-nm irradiance time series of the SBUV/2 instrument onboard the NOAA-9 satellite. They found this time series to differ substantially and qualitatively on these time scales from that of the $\mathrm{Mg}$ II index produced by the same instrument.

The model used to separate the contributions of the centerto-limb behavior and two-sidedness is described in Sect. 2; the smoothing function, Fourier analysis, calibration, and observational and instrumental effects are discussed. The analysis of the solar ultraviolet irradiances is presented in Sect. 3, the results and comparisons with WWB in Sect. 4, and comparision with theoretical semi-empirical model atmospheres in Sect. 5. Section 6 presents our conclusions.

\section{The data analysis model}

If the solid angle subtended by the active region is $\Omega(\mu)$, we can write

$$
\begin{aligned}
& C_{\lambda}(\mu)=\left[\Omega(\mu) I_{\lambda}^{\mathrm{e}}(\mu)\right] /\left[\Omega(\mu) I_{\lambda}^{\mathrm{d}}(\mu)\right], \\
& C_{\lambda}(\mu)=E_{\lambda}^{\mathrm{e}}(\mu) /\left[\Omega(\mu) I_{\lambda}^{\mathrm{d}}(\mu)\right]
\end{aligned}
$$

where

$E_{\lambda}^{\mathrm{e}}(\mu)=\Omega(\mu) I_{\lambda}^{\mathrm{e}}(\mu)$

is the excess irradiance of the active region, which is observable with full-disk irradiance monitors like the SUSIM. Lean et al. (1982) and Lean (1988) find that the excess irradiance of active regions is responsible for the rotational variations seen in the solar ultraviolet irradiances, although Worden et al. (1998) find that the contribution of the enhanced network cannot be discounted. This is the basis for the simple two-component model that we have adopted: the signal, $s_{\lambda}(t)$, at each wavelength is given by:

$s_{\lambda}(t)=q_{\lambda}+c_{\lambda} i(t) \star f_{\lambda}(t)$,

where $q_{\lambda}$ and $c_{\lambda}$ are wavelength-dependent constants; $f_{\lambda}(t)$ is a wavelength-dependent smoothing function; $i(t)$ is a wavelength-independent series of impulses (Dirac delta functions); and " $\star$ " indicates convolution. The $n$th impulse in $i(t)$ represents an active region crossing the center of the solar disk at time $t_{n}$ :

$i(t)=\sum_{n} a_{n} \delta\left(t-t_{n}\right)$,

where the constants $a_{n}$ and times $t_{n}$ are independent of wavelength. The signal contributed by the $n$th active region is

$s_{\lambda, n}(t)=c_{\lambda} a_{n} f_{\lambda}\left(t-t_{n}\right)$.

In physical terms, $s_{\lambda}$ is an observable such as an ultraviolet irradiance or the solar radio flux density at wavelength $\lambda$. If it is an irradiance, $q_{\lambda}$ is the integrated irradiance of the underlying 
disk at wavelength $\lambda$ which includes everything that does not contribute to the rotational variations, notably any more slowly varying, widely distributed active network (Skumanich et al. 1984) which is not explicitly modeled. $c_{\lambda} a_{n}$ is the excess irradiance of the $n$th active region at wavelength $\lambda$ at the moment it crosses the center of the solar disk (i.e., $c_{\lambda}$ is excess surface brightness and $a_{n}$ is solid angle). $f_{\lambda}(t)$ is the smoothing function which describes its variation elsewhere on the disk at wavelength $\lambda$, which does not vary during the solar cycle (Petro et al. 1984; Neckel \& Labs 1994; Greve \& Neckel 1996). Each transit of an active region across the solar disk is treated separately with any change during transit described entirely by the smoothing function, since the transit time is much shorter than the evolutionary time scales of months (Gaizauskas et al. 1983; Harvey et al. 1984). Also, $f_{\lambda}(t)$ is zero for $|t| \geq P_{\odot} / 2$.

In the frequency domain, the Fourier transform, $S_{\lambda}(f)$, of the signal is given by:

$S_{\lambda}(f)=q_{\lambda} \delta(f)+c_{\lambda} I(f) F_{\lambda}(f)$,

where $\delta(f)$ is the Dirac delta function at zero frequency; $I(f)$, the Fourier transform of the series of impulses (Eq. (7)); and $F_{\lambda}(f)$, the Fourier transform of the smoothing function. Since the underlying periodicity is $P_{\odot} \cong 27$ days, the dominant responses in the frequency domain occur at harmonics of the corresponding fundamental frequency, $1 / P_{\odot}$, and, in principle, extend to infinite frequencies. Without a priori knowledge of the individual terms in $q_{\lambda}$ and $c_{\lambda} I(0) F_{\lambda}(0)$, separating their contributions to $S_{\lambda}(0)$ is not feasible. Higher harmonics are reduced since the amplitude of $F_{\lambda}(f)$ tends to decrease as $|f|$ increases because $f_{\lambda}(t)$ is confined and because of the presence of noise; at best only the first and second harmonics (frequencies of $1 / P_{\odot}$ and $\left.2 / P_{\odot}\right)$ are significant:

$S_{\lambda}\left(1 / P_{\odot}\right)=c_{\lambda} I\left(1 / P_{\odot}\right) F_{\lambda}\left(1 / P_{\odot}\right)$

$S_{\lambda}\left(2 / P_{\odot}\right)=c_{\lambda} I\left(2 / P_{\odot}\right) F_{\lambda}\left(2 / P_{\odot}\right)$.

Each expression is the product of three terms, including in both cases the unknown constant, $c_{\lambda}$, which can be eliminated by taking the ratio of the two expressions:

$\mathcal{R}_{\lambda}=S_{\lambda}\left(2 / P_{\odot}\right) / S_{\lambda}\left(1 / P_{\odot}\right)$.

Equation (12) (or its square) is the usual endpoint of such analyses (Donnelly et al. 1986; Donnelly \& Puga 1990). One more step, however, is the separation of the ratio into time- and wavelength-dependent terms, $\mathcal{I}$ and $\mathcal{F}_{\lambda}$, respectively:

$\mathcal{R}_{\lambda}=\mathcal{I F}_{\lambda}$,

where the source and filter parameters, $\mathcal{I}$ and $\mathcal{F}_{\lambda}$, are given by:

$\mathcal{I}=I\left(2 / P_{\odot}\right) / I\left(1 / P_{\odot}\right)$

$\mathcal{F}_{\lambda}=F_{\lambda}\left(2 / P_{\odot}\right) / F_{\lambda}\left(1 / P_{\odot}\right)$.

The source parameter, $\mathcal{I}$, depends only upon the time interval analyzed and is a measure of the degree of two-sidedness. Its variation with time is responsible for the amplitude variations in $\mathcal{R}_{\lambda}$ reported by Donnelly \& Puga (1990).
For an idealized model of $i(t)$ as an infinite series of impulses at intervals of $P_{\odot} / 2$, with values of $a_{n}$ alternating between $a_{1}$ and $a_{2}$,

$\mathcal{I}=1 /(1-r), \quad 0 \leq r \leq 1$,

where $r=a_{2} / a_{1}$. The value varies between 1 and $\infty$ for pure single-sided $(r=0)$ and two-sided $(r=1)$ variations, respectively.

The filter parameter, $\mathcal{F}_{\lambda}$, depends only upon the shape of the smoothing function, $f_{\lambda}(t)$, which becomes narrower as limb darkening increases. An idealized case, which has been applied artificially by Ward $\&$ Shapiro $(1961,1962)$ and Shapiro (1965a), is for $f_{\lambda}(t)$ to be modeled as a rectangle function,

$f_{\lambda}(t)=\Pi(t / T)$,

where $T$ is the full width at half maximum. The rectangle function is described by:

$\Pi(x)= \begin{cases}1, & |x|<1 / 2 \\ 0, & |x|>1 / 2\end{cases}$

and has the sinc function as its Fourier transform (Bracewell 1965)

$F_{\lambda}(f)=T \operatorname{sinc}(f T)=\frac{T \sin (\pi f T)}{\pi f T}$.

The filter parameter is given by:

$\mathcal{F}_{\lambda}(f)=\frac{\sin \left(2 \pi T / P_{\odot}\right)}{2 \sin \left(\pi T / P_{\odot}\right)}$,

which varies between 1 as $T \rightarrow 0$ and 0 for $T=P_{\odot} / 2$. These limits are appropriate for features confined to the solar disk and correspond to extreme limb darkening and to the optically thin case, respectively.

\subsection{The smoothing function}

In analogy to the center-to-limb coefficients used to describe the behavior of the surface brightness of the quiet disk, $L_{\lambda}(\mu)=$ $I_{\lambda}(\mu) / I_{\lambda}(1)$, similar coefficients can be used to describe the center-to-limb behavior of the excess irradiance of an active region:

$\mathcal{L}_{\lambda}^{\mathrm{e}}(\mu)=E_{\lambda}^{\mathrm{e}}(\mu) / E_{\lambda}^{\mathrm{e}}(1)$.

At ultraviolet wavelengths, active regions are essentially confined to the solar disk (Donnelly et al. 1982, 1986; Donnelly \& Puga 1990) and their subtended solid angles are subject to projection, i.e., $\Omega(\mu)=\mu \Omega(1)$. Therefore, the center-to-limb coefficients that describe excess irradiance and surface brightness are related by:

$\mathcal{L}_{\lambda}^{\mathrm{e}}(\mu)= \begin{cases}\mu L_{\lambda}^{\mathrm{e}}(\mu), & 0 \leq \mu \leq 1, \\ 0, & \text { otherwise }\end{cases}$

In accordance with the physical interpretation of Eq. (8),

$c_{\lambda}=I_{\lambda}^{\mathrm{e}}(1)$,

$a_{n}=\Omega_{n}(1)$, 
and the center-to-limb behavior of the excess irradiance can be identified with the smoothing function, $f_{\lambda}(t)$, so the limb brightening and darkening of the $n$th solar active region is described by:

$f_{\lambda}\left(t-t_{n}\right)=\mathcal{L}_{\lambda}^{\mathrm{e}}\left(\mu_{n}\right)$.

The cosine of the heliographic angle, $\mu_{n}$, depends upon the time varying central meridian distance, $\phi_{n}$, of the $n$th active region, on that region's solar latitude, $l_{n}$, and upon the heliographic latitude of the sub-earth point, $B_{0}$ :

$\mu_{n}(t)=\cos \theta_{n}=\cos B_{0} \cos l_{n} \cos \phi_{n}(t)+\sin B_{0} \sin l_{n}$.

The right-hand side of Eq. (25) depends upon time through its implicit dependence on $B_{0}$ and $\phi_{n}$. For the latter, we have:

$\phi_{n}(t)= \begin{cases}2 \pi\left(t-t_{n}\right) / P_{\odot}, & -P_{\odot} / 2 \leq t-t_{n} \leq P_{\odot} / 2, \\ \text { undefined, } & \text { otherwise, }\end{cases}$

where the synoptic period of the Sun, $P_{\odot}$, is nominally 27 days. $B_{0}$ varies with time slowly, having a period of one year and a range of $\pm 7.25^{\circ}$. Our model assumes an active region moves in a straight line across the solar disk, through the center, as the sun rotates (i.e., $l_{n}=B_{0}=0$ ); the effects of these assumptions are examined later.

Many functional forms have been used to describe the center-to-limb behavior (cf. Greve \& Neckel 1996; Hestroffer 1997) but few depend upon a single parameter. We will describe our results using two functional forms that vary more smoothly than the rectangle function. (1) To describe limb brightening and for ease of comparison with WWB, we use their polynomial form multiplied by $\mu$ as in Eq. (22):

$\mathcal{L}_{\lambda}^{\mathrm{e}}(\mu)=\left\{\begin{array}{lr}\mu \max \left[R_{\mathrm{c}}+2\left(1-R_{\mathrm{c}}\right)\left(\mu-\mu^{2} / 2\right), 0\right], \\ 0 \leq \mu \leq 1, \\ 0, & \text { otherwise. }\end{array}\right.$

The surface-brightness ratio, $R_{\mathrm{c}}$, is the parameter that describes the model and is the ratio of the surface brightness at the limb to the surface brightness at the center of the disk. The maximum function is used to avoid negative values when $R_{\mathrm{c}}<0$; otherwise such models could not physically describe strong limb darkening. (2) To describe limb darkening and following Hestroffer (1997) and Hestroffer \& Magnan (1998), we use a truncated power law in $\mu$ characterized by the exponent, $x$ :

$\mathcal{L}_{\lambda}^{\mathrm{e}}(\mu)= \begin{cases}\mu^{x}, & 0 \leq \mu \leq 1, \\ 0, & \text { otherwise. }\end{cases}$

Smoothing functions of both types are shown in the top panel of Fig. 1 for three values of $\mathcal{F}_{\lambda}=0,0.424$, and 0.85 . The curves for constant surface brightness $\left(\mathcal{F}_{\lambda}=0.424, R_{\mathrm{c}}=1\right.$, and $\left.x=1\right)$ are identical. The other two examples illustrate how very different smoothing functions can have the same filter parameter. The case $\mathcal{F}_{\lambda}=0\left(R_{\mathrm{c}}=7.4\right.$ or $\left.x=0\right)$ corresponds to very strong limb brightening; in particular, $x=0$ is the optically thin case, corresponding to a boxcar with a width of 13.5 days (for $P_{\odot}=27$ days). Negative values of $\mathcal{F}_{\lambda}$ occur for larger values of $R_{\mathrm{c}}$ when the excess irradiances peak near the limb,
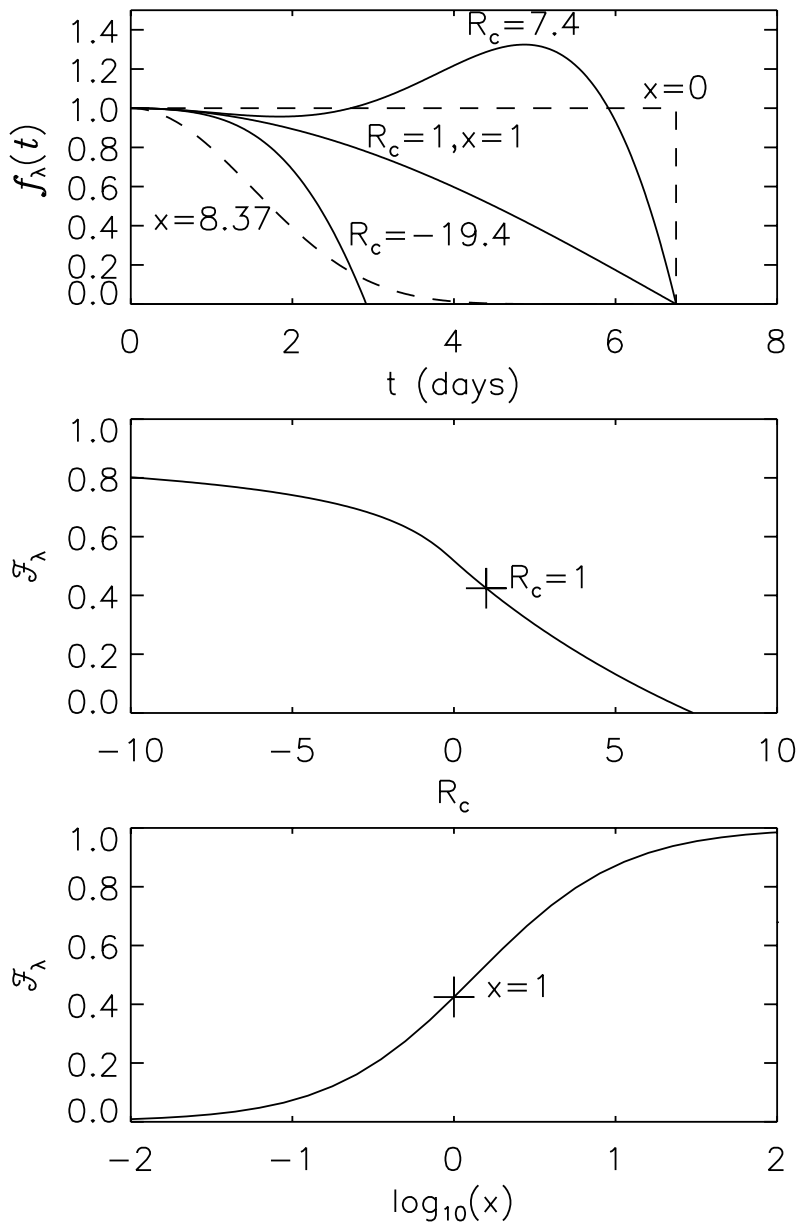

Fig. 1. Model smoothing functions (top) for $R_{\mathrm{c}}$ (solid lines) and $\mu^{x}$ (dotted lines) models and assuming $P_{\odot}=27$ days; the constant curves are identical $\left(R_{\mathrm{c}}=1, x=1, \mathcal{F}_{\lambda}=0.424\right)$ and the other two pairs have the same filter parameter $\left(R_{\mathrm{c}}=-19.4, x=8.37, \mathcal{F}_{\lambda}=0.85\right.$ and $R_{\mathrm{c}}=7.4, x=0, \mathcal{F}_{\lambda}=0$ ). The relationships between $\mathcal{F}_{\lambda}$ and $R_{\mathrm{c}}$, and $\mathcal{F}_{\lambda}$ and $x$, are shown in the middle and bottom panels.

not on the meridian; such behavior would occur for negative $x$ but would have a singularity at the limb. The case $\mathcal{F}_{\lambda}=0.85$ $\left(R_{\mathrm{c}}=-19.4\right.$ or $\left.x=8.37\right)$ corresponds to strong limb darkening; the limiting case is $\mathcal{F}_{\lambda}=1, x=\infty$, an impulse at the origin. The relationships between $R_{\mathrm{c}}$ and $\mathcal{F}_{\lambda}$, and $x$ and $\mathcal{F}_{\lambda}$, are shown in the middle and bottom panels of Fig. 1. A classification system for the limb brightening and darkening of active regions is presented in Table 1 for the range $[0,1]$ of $\mathcal{F}_{\lambda}$. For each value of $\mathcal{F}_{\lambda}$, the corresponding values of the surface-brightness ratio, $R_{\mathrm{c}}$, and the exponent, $x$, are given. The Table also includes the full width at half maximum, $T$, of $\mu^{x}$, assuming $P_{\odot}=27$ days.

\subsection{Calibration}

If the ratio, $\mathcal{R}_{\lambda}$, and filter parameter, $\mathcal{F}_{\lambda}$, are known, the source parameter, $\mathcal{I}$, is easily derived. In the episode under study, the ratios, $\mathcal{R}_{\lambda}$, are known for two common solar indices - the international sunspot number, $R$, and the solar radio flux density, $F_{10.7 \mathrm{~cm}}$ (Crane 1998). As discussed below, the smoothing functions, $f_{\lambda}(t)$, and the filter parameters, $\mathcal{F}_{\lambda}$, are known empirically for both indices. Therefore, as illustrated in Table 2, 
Table 1. Limb brightening and darkening classification for active regions.

\begin{tabular}{ccccl}
\hline \hline $\mathcal{F}_{\lambda}$ & \multicolumn{1}{c}{$R_{\mathrm{c}}$} & $x$ & $T($ dy) & Classification \\
\hline 0.000 & 7.40 & 0.00 & 13.50 & Very strong limb brightening \\
0.110 & 5.36 & 0.16 & 13.40 & Strong limb brightening \\
0.220 & 3.63 & 0.37 & 12.20 & Medium limb brightening \\
0.330 & 2.13 & 0.66 & 10.40 & Weak limb brightening \\
0.424 & 1.00 & 1.00 & 9.00 & Constant surface brightness \\
0.550 & -0.33 & 1.72 & 7.27 & Weak limb darkening \\
0.700 & -3.21 & 3.39 & 5.36 & Medium limb darkening \\
0.850 & -19.41 & 8.37 & 3.46 & Strong limb darkening \\
1.000 & $\infty$ & $\infty$ & 0.00 & Very strong limb darkening \\
\hline
\end{tabular}

Table 2. Calibration of source parameter from $R$ and $F_{10.7 \mathrm{~cm}}$.

\begin{tabular}{|c|c|c|c|c|c|}
\hline Index & $f_{\lambda}(t)$ & $T(\mathrm{dy})$ & $\mathcal{F}_{\lambda}$ & $\mathcal{R}_{\lambda}$ & $\mathcal{I}$ \\
\hline$R$ & $\begin{array}{l}\text { cubic } \\
\text { spline }\end{array}$ & 10.3 & 0.380 & $\begin{array}{l}1.20 \\
\pm 0.09\end{array}$ & $\begin{array}{l}3.15 \\
\pm 0.23\end{array}$ \\
\hline$F_{10.7 \mathrm{~cm}}$ & $\begin{array}{l}\text { truncated } \\
\text { Gaussian }\end{array}$ & 11.1 & 0.158 & $\begin{array}{l}0.48 \\
\pm 0.04\end{array}$ & $\begin{array}{l}3.01 \\
\pm 0.27\end{array}$ \\
\hline $\begin{array}{l}\text { Weighted } \\
\text { mean }\langle\mathcal{I}\rangle\end{array}$ & - & - & - & - & $\begin{array}{l}3.10 \\
\pm 0.07\end{array}$ \\
\hline
\end{tabular}

two independent values for $\mathcal{I}$ can be derived and combined to produce a weighted average, which in turn calibrates Eq. (13) during this episode.

It is then straightforward to obtain the filter parameters, $\mathcal{F}_{\lambda}$, from the measured ratios, $\mathcal{R}_{\lambda}$, at ultraviolet wavelengths. This procedure is comparable to the calibration procedures used in radio, infrared, optical, and speckle interferometry (Coulman 1985; Thompson et al. 1986; Shao \& Colavita 1992).

It is well known that small sunspots become difficult to distinguish as they approach the solar limb. That effect has been quantified by Donnelly et al. (1984) based upon 13432 sunspot counts from 1976 through 1981 . The results are tabulated as a function of central meridian distance and directly measure the smoothing function. For our use, the results have been fit with a cubic spline, with full width at half maximum, $T$ (assuming $P_{\odot}=27$ days), and filter parameter, $\mathcal{F}_{\lambda}$, tabulated in Table 2. The latter value indicates weak limb brightening. Solar emissions with a coronal component - i.e., the solar radio emission at $\lambda 10.7 \mathrm{~cm}$ or soft X-rays $(\lambda \lambda 1-8 \AA)$ - are not limited to the solar disk. In the case of the solar radio flux density, $F_{10.7 \mathrm{~cm}}$, a truncated Gaussian,

$f(t)= \begin{cases}\mathrm{e}^{-4(\ln 2)[t / T]^{2}}, & -P_{\odot} / 2 \leq t \leq P_{\odot} / 2, \\ 0, & \text { otherwise }\end{cases}$

with full width at half maximum, $T$, of 11.1 days (for $P_{\odot}=$ 27 days), appears to be a good match, as estimated from Donnelly \& Puga (1990, Fig. 7) and summarized in Table 2. The filter parameter, $\mathcal{F}_{\lambda}$, indicates strong limb brightening.
Table 3. Effects of solar latitude and polar tilt on the filter parameter. For all cases, $f_{\lambda}(t)=\mu$.

\begin{tabular}{rccl}
\hline \hline$l\left(^{\circ}\right)$ & $B_{0}\left({ }^{\circ}\right)$ & $\mathcal{F}_{\lambda}$ & Comment \\
\hline 0 & 0 & 0.424 & Constant \\
0 & 7.25 & 0.424 & No change \\
20 & 0 & 0.424 & No change \\
40 & 0 & 0.424 & No change \\
20 & 7.25 & 0.400 & Apparent weak limb brightening \\
20 & -7.25 & 0.450 & Apparent weak limb darkening \\
40 & 7.25 & 0.367 & Apparent weak limb brightening \\
40 & -7.25 & 0.483 & Apparent weak limb darkening \\
\hline
\end{tabular}

\subsection{Observational and instrumental effects}

As noted earlier, the model described above assumes that an active region moves in a straight line along the solar equator through the sub-Earth point. It also assumes that the sensitivity of the measuring instrument to an active region does not depend on its position on the solar disk.

The first assumption is untrue. Active regions are distributed over a wide range of heliographic latitudes throughout a solar cycle, even at the same time - e.g., the "butterfly diagram" of Maunder (1922). The solar latitudes of individual active regions are appropriately constant. The dependence of $\mu_{n}$ upon the solar latitude of the active region and the heliographic latitude of the sub-earth point, as well as the central meridian distance, is apparent in Eq. (26), but previous calculations have assumed that both $l_{n}$ and $B_{0}$ are zero. The effects on the filter parameter of nonzero $l_{n}$ and $B_{0}$ have been calculated for constant surface brightness using Eqs. (26) and (27) for several values of $l_{n}$ and $B_{0}$, with the predicted filter parameters presented in Table 3. During the time interval under study, active regions predominantly were located at latitudes between $\pm 20^{\circ}$ and $B_{0}$ varied over approximately three quarters of its annual cycle (with a maximum amplitude of $7.25^{\circ}$ ). The maximum changes in the filter parameter are approximately \pm 0.03 , which arise when both $l_{n}$ and $B_{0}$ are nonzero. The offset in $\mu_{n}$ introduced by the second term on the right hand side of Eq. (26) effectively changes the width of the smoothing function by a small amount. When averaged over active regions at many different latitudes and over a significant fraction of an annual cycle, the net effect will be even smaller and not significant to this study.

Of potentially greater consequence is an instrumental effect, the variation in sensitivity or response across the field of view (FOV) of a full-disk spectrometer like the SUSIM. Ground-based calibrations provide only weak constraints on the FOV effect because spatially dependent in-flight degradation of the primary grating caused by UV exposure quickly dominates the pre-launch response (Floyd et al. 1998a). In space, the response can be measured using offset scans of the sun as a function of wavelength, and the corrections derived can be applied to the measurements. But the measured response is averaged over the solar disk, and, so, does not measure the response to an individual active region as it moves across the field 
Table 4. Effect of the instrumental field of view (FOV) response ratio (center to limb) on the filter parameter. For all cases, $f_{\lambda}(t)=\mu$.

\begin{tabular}{lcl}
\hline \hline $\begin{array}{c}\text { FOV } \\
\text { ratio }\end{array}$ & $\mathcal{F}_{\lambda}$ & $\begin{array}{l}\text { Description of apparent limb } \\
\text { brightening or darkening }\end{array}$ \\
\hline 0.9 & 0.444 & Weak limb darkening \\
1 & 0.424 & No brightening or darkening \\
1.1 & 0.406 & Weak limb brightening \\
1.4 & 0.355 & Weak limb brightening \\
2.0 & 0.272 & Medium limb brightening \\
3.0 & 0.170 & Strong limb brightening \\
4.0 & 0.097 & Very strong limb brightening \\
\hline
\end{tabular}

of view. The difference between the two responses will act as an artificial source of limb brightening or darkening.

The orientation of the spectrometer is fixed in the spacecraft reference frame, but varies with respect to the heliocentric reference frame. The relation between these reference frames is unknown without greater knowledge of the spacecraft orbit and attitude than is readily available. Consequently, because of this variation the effect of any linear term in the FOV response will be reduced, and a quadratic term will cause a systematic apparent limb brightening (positive) or limb darkening (negative). The magnitude of the effect is estimated in Table 4 for several values of the point-source response (circularly symmetric) at the solar limb, normalized to the value at sun center, for constant surface brightness. For the most extreme example where the FOV response ratio equals 4, apparent very strong limb brightening is observed. As will be discussed below, such large effects are characteristic of the SUSIM in the vicinity of the Ly- $\alpha$ emission line (121.6 nm). At longer wavelengths the linear and quadratic terms are both factors of 10-100 times smaller. The potential uncertainties in $\mathcal{F}_{\lambda}$ are \pm 0.02 and not significant to this study.

\section{Analysis}

The time interval chosen for study, 1994 September 27 to 1995 June 4, is an episode (Crane 1998) near the end of solar cycle 22 of persistent $\sim 13.5$-day variations in the time series for the international sunspot number, $R$. The time series and clean Fourier transforms during this episode for $R$ and for $F_{10.7 \mathrm{~cm}}$ are reproduced in Fig. 2. The corresponding subset (UARS days 1112-1362) of the Version 19 Level-3BS 1-nm gridded, 1-nm integrated ultraviolet irradiances measured by the SUSIM also exhibits 13.5-day variations (Floyd et al. 1998b) and has been analyzed with the same DFT/CLEAN technique used by Crane (1998), which combines the direct Fourier transform (DFT) and the CLEAN deconvolution algorithm (Roberts et al. 1987; Roberts \& Lehár 1994; Crane 2001). The most recent version of the SUSIM irradiance data is freely available ${ }^{1}$. The amplitudes of the clean Fourier transforms as a function of period and wavelength are shown as a gray-scale plot in Fig. 3. Because the solar irradiances at these wavelengths vary by about five orders of magnitude, the Fourier transform at each wavelength

\footnotetext{
${ }^{1}$ URL: ftp://susim.nrl.navy.mil/pub/uars
}
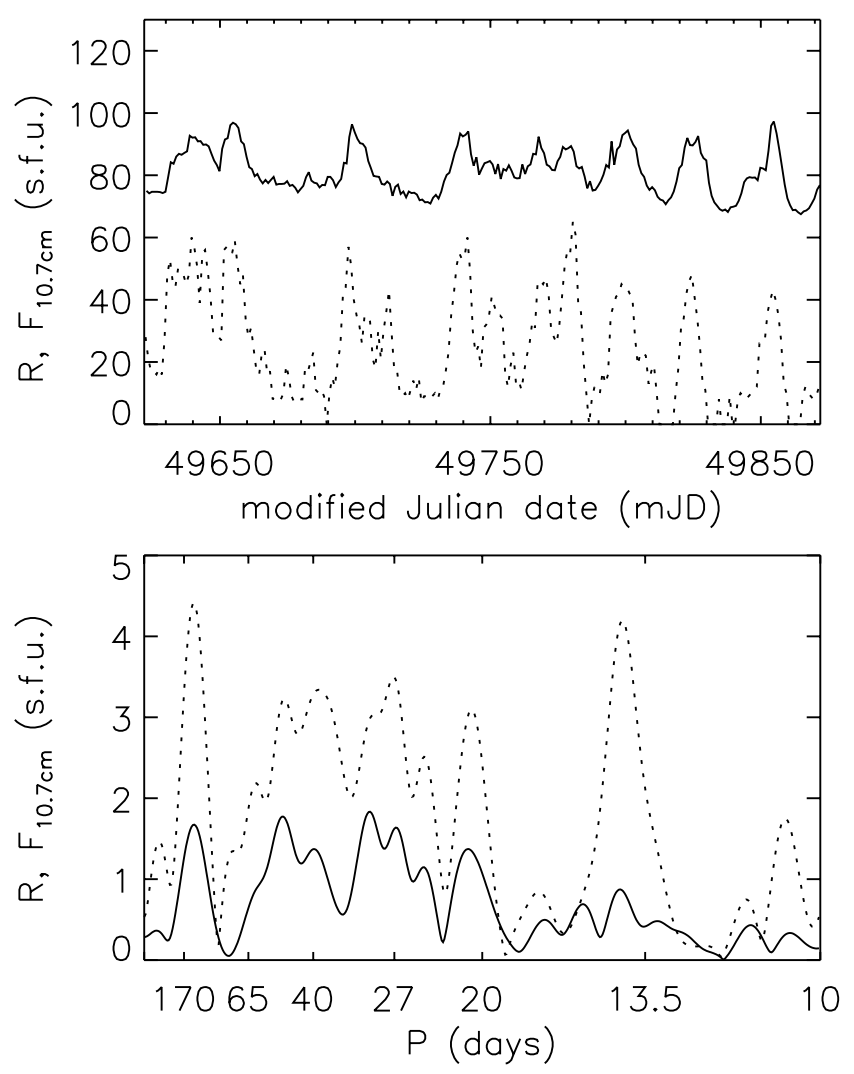

Fig. 2. Time series (top) and amplitudes of the clean Fourier transforms (bottom) as a function of period, $P$, for the international sunspot number, $R$ (dotted line), and solar radio flux density, $F_{10.7 \mathrm{~cm}}$ (solid line), during the episode of $\sim 13.5$-day variations during solar cycle 22 (based upon Crane 1998).

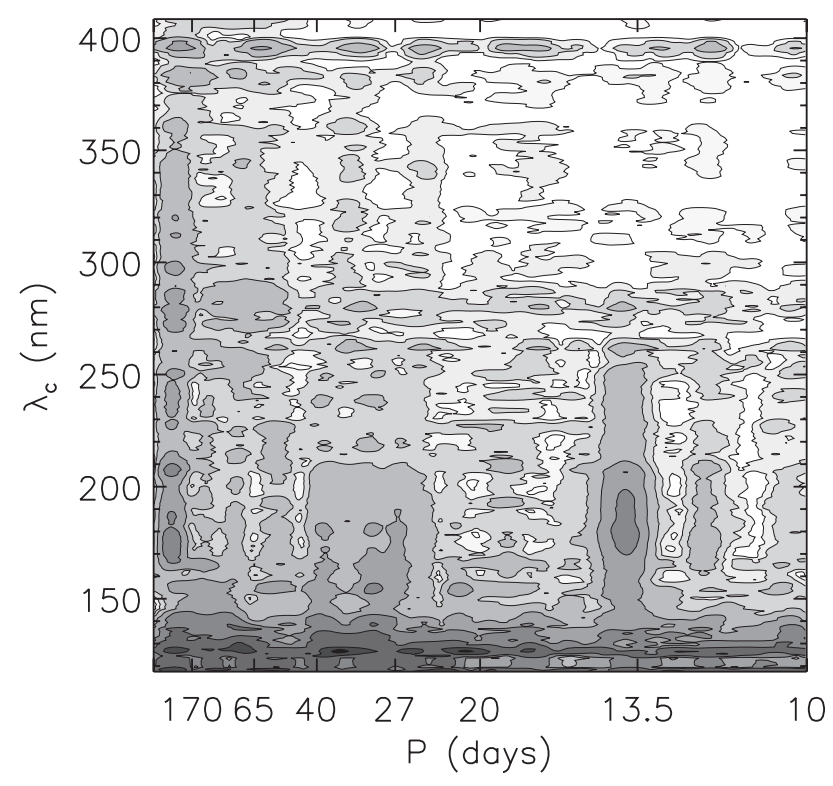

Fig. 3. Contour plot of the amplitudes of the clean Fourier transforms for the 1-nm binned, 5-nm smoothed ultraviolet irradiances measured by the SUSIM for the interval 1994 September 27 to 1995 June 4. The ordinate is the central wavelength, $\lambda_{\mathrm{c}}$, of the 5 -nm windows, and the abscissa is the period, $P$. The values at each wavelength are normalized to the corresponding DC value at zero frequency. The grey-scale and contour levels correspond to $(0.005,0.01,0.02,0.05,0.1,0.2,0.5,0.9)$ of the peak normalized value of 4.44 . 


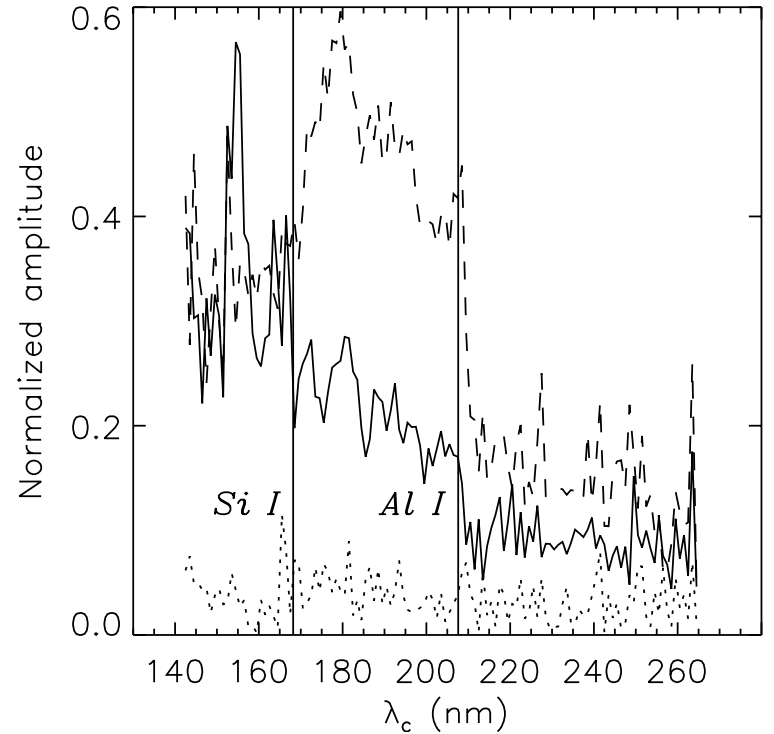

Fig. 4. Normalized peak amplitudes of the $\sim 27$-day (solid line) and $\sim 13.5$-day (dashed line) periodicities measured from the 1-nm gridded ultraviolet irradiances measured by the SUSIM for the interval 1994 September 27 to 1995 June 4 . The windows searched for the peaks were $25-30$ days and 12.5-15 days, respectively. The abscissa is the central wavelength, $\lambda_{\mathrm{c}}$, of the 1-nm bins. Vertical lines indicate the $\mathrm{Si}$ I edge at $168.2 \mathrm{~nm}$ and the $\mathrm{Al} \mathrm{I}$ edge at $207.6 \mathrm{~nm}$. The dotted line indicates the estimated errors based upon Eq. (31).

has been normalized by its value at zero frequency, the "DC" value. To reduce the noise in Fig. 3, the normalized amplitudes have been smoothed in wavelength with a 5-nm boxcar. While other periodicities are visible, those of interest at $\sim 27$ days and $\sim 13.5$ days are significant in the wavelength interval 142$265 \mathrm{~nm}$. No significant $\sim 27$-day or $\sim 13.5$-day variations are seen at shorter and longer wavelengths because of high noise and low signal, respectively, except at Ly- $\alpha(121.6 \mathrm{~nm})$ and $\mathrm{Mg}$ II $(279.9 \mathrm{~nm})$. Individual analyses have been performed for the SUSIM Ly- $\alpha$ and core-to-wing Mg II indices.

The peak amplitudes in two windows covering periods of 25-30 days and 12.5-15.0 days in the normalized clean Fourier transform at each wavelength have been determined. The rootmean-squared (rms) error at each wavelength is estimated from the mean amplitude at high frequencies (periods of 2-10 days). In the absence of signals, these amplitudes are described by a Rayleigh distribution, for which

$\langle A\rangle=(\pi / 2)^{1 / 2} \sigma$.

This equation can be inverted and used to estimate the error from the mean amplitude at high frequencies.

The peak amplitudes of the $\sim 27$-day and $\sim 13.5$-day periodicities obtained for the wavelength interval 142-265 nm are shown in Fig. 4. Three regions are easily distinguished, separated by the Si I edge at $168.2 \mathrm{~nm}$ and by the Al I edge at $207.6 \mathrm{~nm}$ (where the height of formation of the solar continuum abruptly changes, as photo-ionization from the $\mathrm{Si} \mathrm{I}{ }^{1} \mathrm{D}$ $(168.2 \mathrm{~nm})$ or the $\mathrm{Al} \mathrm{I}{ }^{2} \mathrm{P}^{0}(207.6 \mathrm{~nm})$ energy levels discontinuously becomes the dominant term in the continuous opacity at shorter wavelengths). In the region below the Si I edge, the amplitudes of the $\sim 27$-day and $\sim 13.5$-day periodicities are

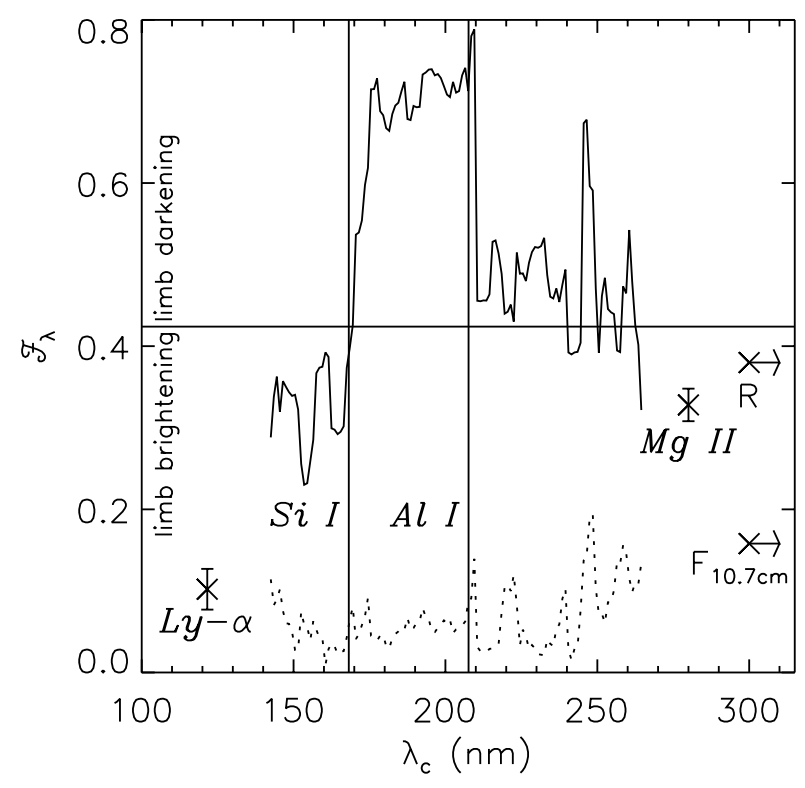

Fig. 5. Filter parameters, $\mathcal{F}_{\lambda}$, calculated from the weighted means, using a 5-nm boxcar, of the ratios, $\mathcal{R}_{\lambda}$, of the amplitudes of the $\sim 13.5$-day and $\sim 27$-day periodicities (shown in Fig. 4) and the mean source parameter, $\langle\mathcal{I}\rangle$, from Table 2. Shown are the theoretical filter parameters for the international sunspot number, $R$, and solar radio flux density, $F_{10.7 \mathrm{~cm}}$, and the measured values for the SUSIM Ly- $\alpha$ irradiance and the Mg II core-to-wing ratio index. The abscissa is the central wavelength, $\lambda_{\mathrm{c}}$, of the 5-nm windows. Vertical lines indicate the Si I edge at $168.2 \mathrm{~nm}$ and the $\mathrm{Al} \mathrm{I}$ edge at $207.6 \mathrm{~nm}$. The dotted line indicates the estimated errors derived from the errors in the amplitudes.

approximately constant and equal. In the region above the Al I edge, the amplitudes of the $\sim 27$-day and $\sim 13.5$-day periodicities are approximately constant and the latter are twice the former. Between the Si I and Al I edges the amplitudes of the $\sim 27$-day periodicities decline approximately linearly from the values at the short wavelengths to those at the long wavelengths. On the other hand, the amplitudes of the $\sim 13.5$-day periodicities increase by about a factor of 2 across the Si I edge and decrease by about a factor of 1.5 across the Al I edge.

The ratios, $\mathcal{R}_{\lambda}$, are readily calculated from these measurements. The next step, new to this analysis, is to proceed as described in Sect. 2.2 using the weighted average source parameter, $\langle\mathcal{I}\rangle$, from Table 2. Despite the factor of $\sim 2.5$ difference between the ratios for the international sunspot number, $R$, and the solar radio flux density, $F_{10.7 \mathrm{~cm}}$, the two estimates of the source parameter are in excellent agreement. The average value of 3.1 indicates significant two-sidedness since $r=0.68$ in the idealized model of Eq. (16). The calibrated values of the filter parameters, $\mathcal{F}_{\lambda}$, are shown in Fig. 5. The three regions separated by the $\mathrm{Si}$ I and $\mathrm{Al}$ I edges are apparent; the transition at the Si I edge occurs gradually over the interval $168-175 \mathrm{~nm}$ but the transition at the $\mathrm{Al}$ I edge occurs abruptly at $210 \mathrm{~nm}$; these transitions were apparent in the earlier work with the ratios, $\mathcal{R}_{\lambda}$ (Donnelly \& Puga 1990). Table 5 includes weighted averages of $\mathcal{F}_{\lambda}$ for the wavelength intervals $142-168 \mathrm{~nm}, 175-210 \mathrm{~nm}$, and $210-265 \mathrm{~nm}$ and for the Ly- $\alpha$ irradiance and the Mg II index. The average values of the filter parameters have been used for classification and to derive values of $R_{\mathrm{c}}, x$, and $T$. 
Table 5. Means of measured filter parameters, $\mathcal{F}_{\lambda}$, and classifications for active regions.

\begin{tabular}{ccclrrr}
\hline \hline$\lambda \lambda(\mathrm{nm})$ & $\left\langle\mathcal{R}_{\lambda}\right\rangle$ & $\left\langle\mathcal{F}_{\lambda}\right\rangle$ & Classification & $R_{\mathrm{c}}$ & \multicolumn{1}{c}{$x$} & $T(\mathrm{dy})$ \\
\hline Ly- $\alpha$ & $0.32 \pm 0.08$ & $0.102 \pm 0.025$ & Very strong limb brightening & 5.50 & 0.15 & 13.4 \\
$142-168$ & $1.09 \pm 0.18$ & $0.352 \pm 0.060$ & Weak limb brightening & 1.85 & 0.73 & 10.1 \\
$175-210$ & $2.20 \pm 0.18$ & $0.710 \pm 0.060$ & Strong limb darkening & -3.58 & 3.56 & 5.2 \\
$210-265$ & $1.39 \pm 0.23$ & $0.448 \pm 0.073$ & Weak limb darkening & 0.74 & 1.12 & 8.7 \\
Mg II & $1.02 \pm 0.06$ & $0.328 \pm 0.020$ & Medium limb brightening & 2.16 & 0.66 & 10.5 \\
\hline
\end{tabular}

\section{Results and comparisons}

Few measurements of the center-to-limb behavior of active regions at ultraviolet wavelengths are available for comparison with the SUSIM results presented above. WWB has used SOLSTICE data to study the behavior of multiple components, including active regions and the quiet disk, in the wavelength range $120-170 \mathrm{~nm}$. Information on the behavior of the quiet disk at ultraviolet wavelengths is available from Basri et al. (1979, 121.6 nm), Roussel-Dupré (1982, 121.6 nm), Brekke \& Kjeldseth-Moe (1994b, 144.3-168.2 nm), Samain (1979, 146.0-209.8 nm), Kjeldseth Moe \& Milone (1978, 194.5$324.5 \mathrm{~nm}$ ), and Bonnet (1968, 196.6-283.9 nm); unfortunately, the sparse wavelength sampling of the results from SUMER (Wilhelm et al. 1998; Dammasch et al. 1999) only marginally overlaps with the wavelength range of the SUSIM results. When we combine these results, we can describe the centerto-limb behavior of solar active regions and the solar contrast throughout the far and mid ultraviolet (100-200 nm and 200-300 nm).

\subsection{The Ly- $\alpha$ emission line}

The measured filter parameter for the Ly- $\alpha$ irradiance indicates very strong limb brightening for active regions which disagrees with the weak limb darkening $\left(R_{\mathrm{c}}=0.77\right)$ found in WWB and predicted by Fontenla et al. (1999), as well as the weak limb brightening for the quiet disk seen by Basri et al. (1979) and Roussel-Dupré (1982). The Ly- $\alpha$ spectroheliograms in Prinz (1974) also indicate little change in surface brightness or contrast for active regions distributed across the solar disk and even near the limb.

The most likely explanation for the discrepancy in the SUSIM result is the instrumental effect discussed in Sect. 2.3 the field-of-view (FOV) response of the instrument which exhibits its greatest variation at the shortest wavelengths, in the vicinity of Ly- $\alpha$. The size of the effect has been illustrated in Table 4. A center-to-limb FOV response ratio of 2 approximates that of SUSIM at Ly- $\alpha$ when first measured in November 1995. The very strong limb brightening implied by the observed filter parameter could be explained if the value of this ratio were 4 , which, given the difficulty of the measurements, cannot be excluded.

\subsection{The interval $142-168 \mathrm{~nm}$}

In the wavelength range $142-168 \mathrm{~nm}$, the average filter parameter from the SUSIM results for active regions indicates weak

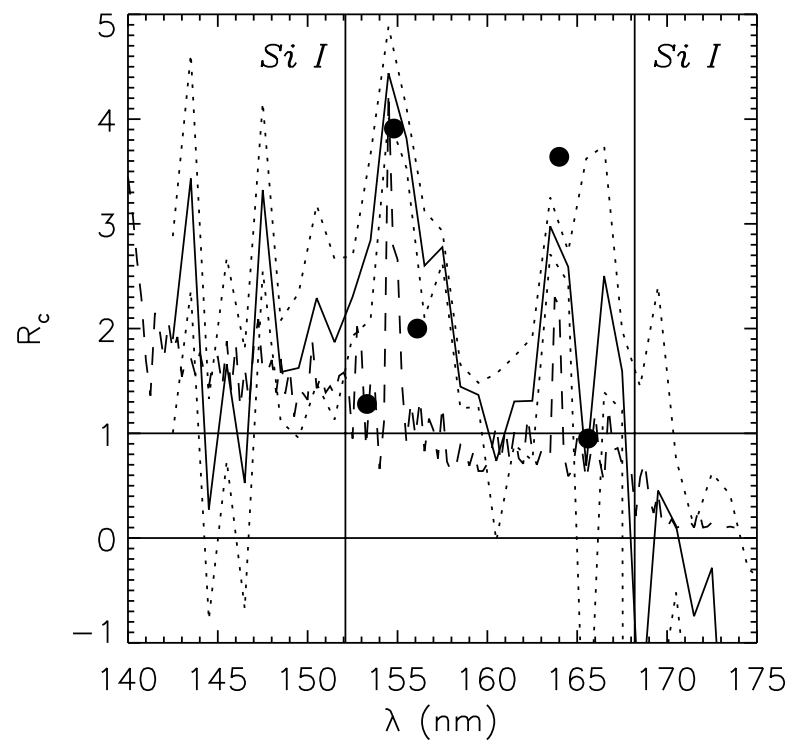

Fig. 6. Surface-brightness ratios, $R_{\mathrm{c}}$, for the wavelength range 140 $175 \mathrm{~nm}$ derived from the filter parameters for the SUSIM 1-nm ratios (solid line) and from WWB for the SOLSTICE plage continuum (dashed line) and emission lines (solid circles). The dotted lines indicate the range of the estimated errors in the SUSIM ratios.

limb brightening. Consequently, in Fig. 6, the filter parameters have been expressed in terms of the corresponding surfacebrightness ratio, $R_{\mathrm{c}}$. The trend is from weak limb brightening at $142 \mathrm{~nm}$ to constant/weak limb darkening at $168 \mathrm{~nm}$ with significant limb-brightened features at $\sim 155 \mathrm{~nm}$ and $\sim 164 \mathrm{~nm}$.

Comparison in Fig. 6 with the plage results obtained by WWB shows excellent agreement after allowance for the different resolutions of the two instruments. The instrument function of the SUSIM is a triangle with a full width at half maximum of $\sim 1.1 \mathrm{~nm}$, while that of the SOLSTICE is roughly rectangular with a width of $0.25 \mathrm{~nm}$; the higher resolution allows WWB to isolate several emission lines. At short wavelengths, below the $\mathrm{Si} \mathrm{I}^{3} \mathrm{P}$ photo-ionization limit at $152.1 \mathrm{~nm}$, the SUSIM results are noisy but agree with those from WWB. At longer wavelengths the agreement is excellent except for the two limbbrightened features seen by the SUSIM. These features can be understood in terms of the lower resolution of the SUSIM and the blending of continuum and emission-line features seen in WWB. The peak of the feature at $\sim 155 \mathrm{~nm}$ coincides with a SOLSTICE continuum feature at $154.50 \mathrm{~nm}$ and the C IV emission line at $154.8 \mathrm{~nm}$, and all three have comparable $R_{\mathrm{c}}$; blending with the $\mathrm{C} I$ emission feature at $156.1 \mathrm{~nm}$ contributes to the width. The second feature at $\sim 164 \mathrm{~nm}$ coincides with a 


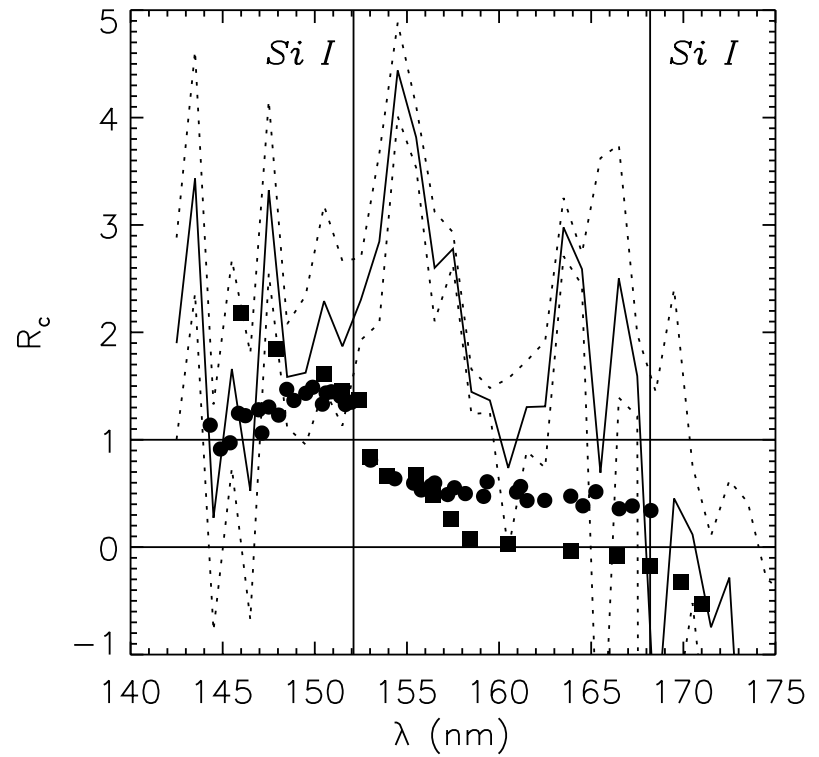

Fig. 7. Surface-brightness ratios, $R_{\mathrm{c}}$, for the wavelength range 140 $175 \mathrm{~nm}$ derived from the filter parameters for the SUSIM 1-nm ratios (solid line) and derived from Brekke \& Kjeldseth-Moe (1994b; solid circles) and Samain (1979; solid squares) for the quiet-disk continuum. The dotted lines indicate the range of the estimated errors in the SUSIM ratios.

SOLSTICE continuum feature at $163.75-164.00 \mathrm{~nm}$ and the He II emission line at $164.0 \mathrm{~nm}$; the observed $R_{\mathrm{c}}$ corresponds to a blend of the two.

The observed behavior of active regions also agrees with that reported by Brekke \& Kjeldseth-Moe (1994b) and Samain (1979) for the quiet-disk continuum shortward of the Si I edge at $152.1 \mathrm{~nm}$ (Fig. 7). However, unlike the transition to limb darkening they see at $152.1 \mathrm{~nm}$, the transition to limb darkening in the SUSIM results is more gradual and starts at the Si I edge at $168.2 \mathrm{~nm}$. Below $152.1 \mathrm{~nm}$, the contrast is approximately constant; above, but still below $168.2 \mathrm{~nm}$, the contrast increases toward the limb.

\subsection{The interval $168-210 \mathrm{~nm}$}

Because of the dominance of limb darkening above $168 \mathrm{~nm}$, the filter parameters have been expressed in terms of the corresponding exponent, $x$, for presentation in Fig. 8. Active regions exhibit a gradual transition to strong limb darkening extending from the Si I edge at $168.2 \mathrm{~nm}$ to about $175 \mathrm{~nm}$. The transition back to weak limb darkening occurs abruptly at $210 \mathrm{~nm}$. Between these limits, the active regions and the quiet-disk continuum (Samain 1979; Kjeldseth Moe \& Milone 1978; Bonnet 1968) exhibit approximately constant strong and weak limb darkening, respectively. The average difference in the exponent, $x$, between the SUSIM results and the continuum measurements of Samain (1979) is $1.83 \pm 0.08$; that is, the center-to-limb behavior of the solar contrast is like $\mu^{1.8}$ over the wavelength range 175-210 $\mathrm{nm}$.

Such behavior, however, disagrees with the results of Hersé (1979) who, from high-resolution photographs of the sun, deduced that at 200 and $210 \mathrm{~nm}$ facular grains exhibit

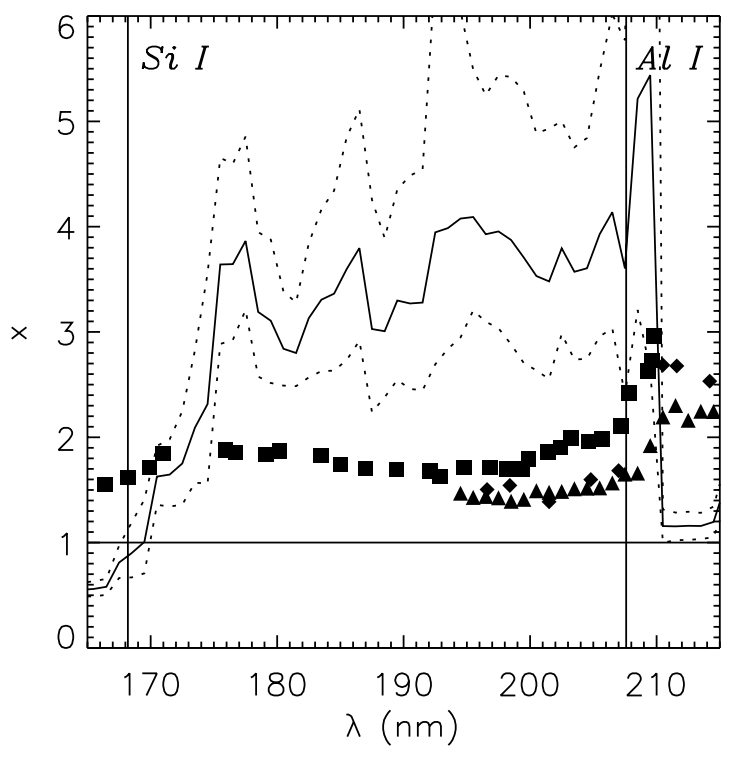

Fig. 8. Exponents, $x$, for the wavelength range $165-215 \mathrm{~nm}$ derived from the filter parameters for the SUSIM 5-nm smoothed ratios (solid line) and derived from Samain (1979; solid squares), Kjeldseth Moe \& Milone (1978; solid triangles), and Bonnet (1968; solid diamonds) for quiet-disk continuum. Over the wavelength range $175-210 \mathrm{~nm}$, the average difference in exponent between the SUSIM and Samain results is $1.83 \pm 0.08$. The dotted lines indicate the range of the estimated errors in the SUSIM exponents.

approximately constant contrast with position on the disk. The discrepancy arises, we believe, because his 200-nm and 210$\mathrm{nm}$ filters had significant transmission on both sides of the Al I edge, allowing contamination by emission longward of the Al I edge where the solar contrast decreases toward the limb (see the next section).

\subsection{The interval $210-265 \mathrm{~nm}$}

Above the Al I edge at $207.6 \mathrm{~nm}$, Kjeldseth Moe \& Milone (1978) and Bonnet (1968) show (Fig. 9) weak or medium limb darkening for the quiet-disk continuum. On the other hand, active regions exhibit only weak, perhaps marginally significant, limb darkening or brightening. Since Greve \& Neckel (1996) report that Kjeldseth Moe \& Milone (1978) underestimate the degree of limb darkening, we have calculated the average difference in the exponent, $x$, between the SUSIM results and the continuum measurements of Bonnet (1968). The result is $-1.36 \pm 0.06$; that is, the center-to-limb behavior of the solar contrast is like $\mu^{-1.4}$ over the wavelength range $210-265 \mathrm{~nm}$. The increase near the limb resembles that of faculae at wavelengths $\geq 330 \mathrm{~nm}$ (Foukal et al. 1991; Unruh et al. 1999).

\subsection{The Mg II index}

The observed medium limb brightening of the Mg II core-towing index indicates (Fig. 5) that the core of the $\mathrm{Mg}$ II absorption feature $(279.9 \mathrm{~nm})$ exhibits less limb darkening than do the wings - by $\Delta \mathcal{F}_{\lambda}$ of $0.10 \pm 0.06$, which agrees with the variation $(\sim 0.09)$ between the line center and its wings for 


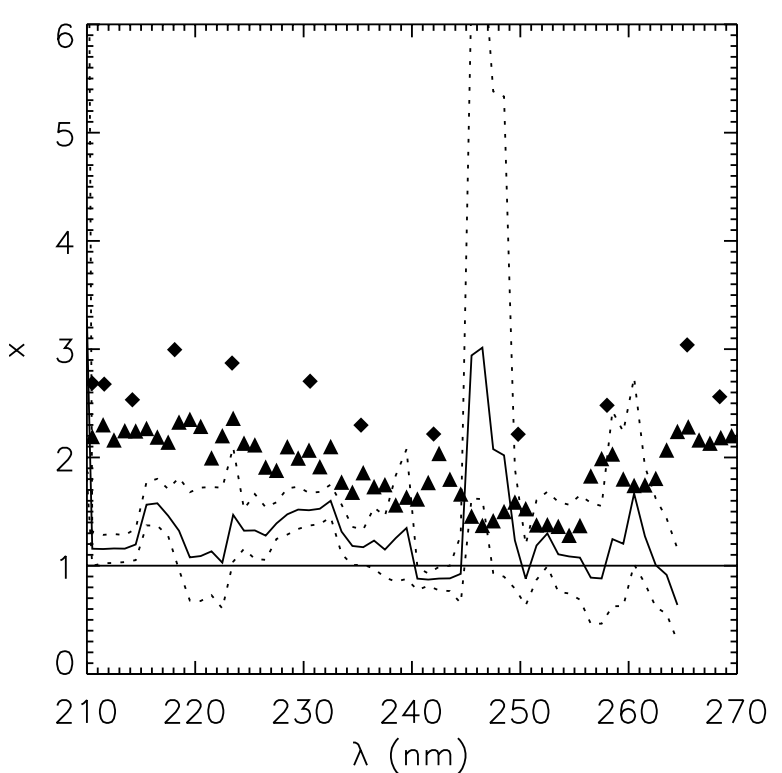

Fig. 9. Exponents, $x$, for the wavelength range 210-270 $\mathrm{nm}$ derived from the filter parameters for the SUSIM 5-nm smoothed ratios (solid line), and derived from Kjeldseth Moe \& Milone (1978; solid triangles) and Bonnet (1968; solid diamonds) for the quiet-disk continuum. Over the wavelength range $210-265 \mathrm{~nm}$, the average difference in exponent between the SUSIM and Bonnet results is $-1.36 \pm 0.06$. The dotted lines indicate the range of the estimated errors in the SUSIM exponents.

the quiet sun, based upon Kjeldseth Moe \& Milone (1978). Unfortunately, even the recent observations of Morrill et al. (2001) include only a single active region and provide no information on center-to-limb behavior.

\section{Comparison with model atmospheres}

Semi-empirical model calculations of the emergent intensity from the solar atmosphere offer further comparisons with the results of our analysis of the SUSIM irradiance observations. Such model calculations provide surface brightness for the quiet sun and active regions for a range of $\mu$ which can be compared with our observed results. The irradiance observations offer additional tests of the models, while improved models can offer a physical understanding of the center-to-limb behavior versus wavelength which we found.

We have used the latest version of the semi-empirical solar model atmospheres described by Fontenla et al. (1999) and extended and improved by one of us (Avrett). Model emergent intensities are calculated at multiple discrete wavelengths and at eight values of $\mu$ for models of a range of solar features, including the faint plage (FP) and the quiet sun (QS) (corresponding to the models $\mathrm{H}$ and C, respectively, of Fontenla et al. 1999). We found the plage $(\mathrm{P})$ model intensities to be far too bright to represent the average active region, and so we calculated the excess surface brightness, $I^{\mathrm{e}}$, according to

$I^{\mathrm{e}}=\mathrm{FP}-\mathrm{QS}$,

(where FP and QS represent the faint plage and quiet sun emergent intensities, respectively) for 156 discrete wavelengths in

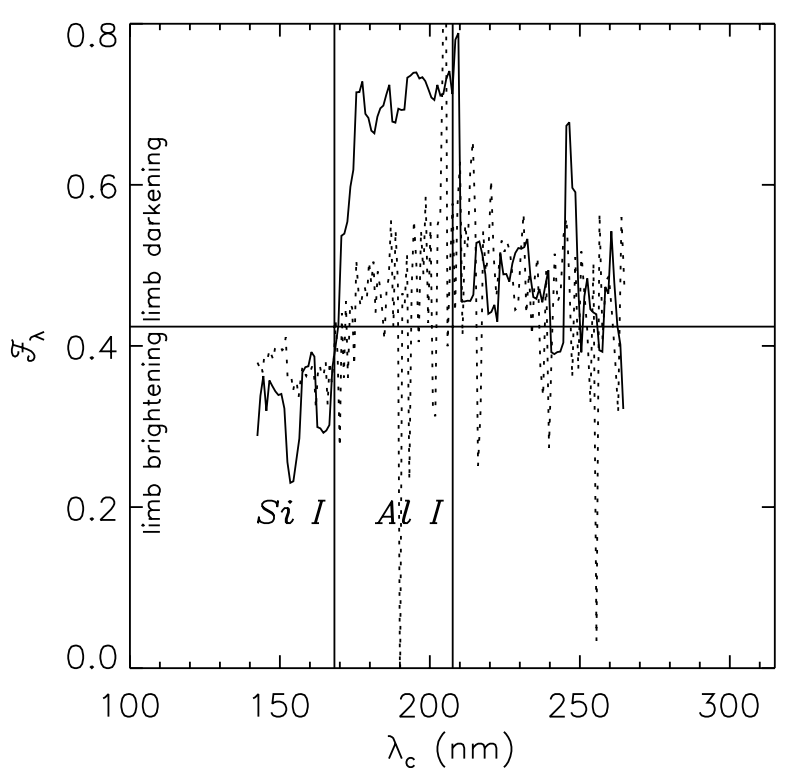

Fig. 10. Filter parameters, $\mathcal{F}_{\lambda}$ (solid) and $\mathcal{F}_{\lambda}^{\text {model }}$ (dotted) for the wavelength range 142-265 nm calculated from the SUSIM 5-nm data in Fig. 5 and the model data of Avrett, respectively. Vertical lines indicate the Si I edge at $168.2 \mathrm{~nm}$ and the $\mathrm{Al}$ I edge at $207.6 \mathrm{~nm}$.

the wavelength range 142-265 $\mathrm{nm}$. Excess irradiances were calculated for $\Omega(1)=1$ according to Eq. (5) using eight values of $\mu(1,0.8,0.6,0.4,0.3,0.2,0.1$, and 0.05$)$, supplemented by the null value at the limb since $\Omega(0)=0$. Each set of discrete values was fit with a cubic spline and the filter parameter calculated. The model-based filter parameters are compared in Fig. 10 to those from the SUSIM measurements shown in Fig. 5.

The model-based filter parameters show greater scatter than do the smoothed SUSIM-based values. This is the result of two factors. The SUSIM observations are integrated over $5 \mathrm{~nm}$, while the model surface brightnesses correspond, in principle, to full resolution at discrete wavelengths. In addition, the model treatment of lines involves: 1) a sampling of 5 representative lines in each $10-\mathrm{nm}$ interval (selecting the 10th to 90th of 100 lines in order of their strength, as described by Avrett, Machado, and Kurucz 1986), and 2) the use of a scattering albedo as a function of height, chosen so that the computed emission lines are in general agreement with observations. Thus we do not expect to match the observed spectrum in detail. The exact values of FP and QS model intensities at the various discrete wavelengths can be artificially noisy.

At wavelengths below $168 \mathrm{~nm}$ and above $210 \mathrm{~nm}$, the good agreement of the model- and SUSIM-based filter parameters is apparent and confirmed by the agreement of the mean filter parameters in Table 6 . In the wavelength range 168-210 nm the poorer agreement is apparent in Fig. 10 and Table 6: the model values exhibit only weak limb darkening instead of the strong limb darkening actually observed. Furthermore, the model-based filter parameters vary more smoothly across the Si I (168.2 nm) and Al I (207.6 nm) edges.

Table 6 also includes separate entries for the QS and FP models and for the measured quiet disk (as cited). This additional information allows us to determine that the FP model 
Table 6. Comparison of mean filter parameters.

\begin{tabular}{ccccccl}
\hline \hline$\lambda \lambda(\mathrm{nm})$ & $\left\langle\mathcal{F}_{\lambda}\right\rangle$ & $\left\langle\mathcal{F}_{\lambda}^{\text {model }}\right\rangle$ & $\left\langle\mathcal{F}_{\lambda}^{\text {model, QS }}\right\rangle$ & $\left\langle\mathcal{F}_{\lambda}^{\text {model, FP }}\right\rangle$ & $\left\langle\mathcal{F}_{\lambda}^{\text {quiet }}\right\rangle$ & Reference for $\mathcal{F}_{\lambda}^{\text {quiet }}$ \\
\hline $142-168$ & $0.352 \pm 0.060$ & $0.372 \pm 0.020$ & $0.402 \pm 0.033$ & $0.379 \pm 0.018$ & $0.435 \pm 0.036$ & Brekke \& Kjeldseth-Moe (1994b) \\
& & & & & $0.444 \pm 0.065$ & Samain (1979) \\
$175-210$ & $0.710 \pm 0.060$ & $0.486 \pm 0.088^{a}$ & $0.530 \pm 0.054$ & $0.516 \pm 0.042$ & $0.577 \pm 0.037$ & Samain (1979) \\
$210-265$ & $0.448 \pm 0.073$ & $0.471 \pm 0.104$ & $0.522 \pm 0.044$ & $0.514 \pm 0.048$ & $0.536 \pm 0.033$ & Kjeldseth Moe \& Milone (1978) \\
& & & & $0.642 \pm 0.023$ & Bonnet (1968) \\
\hline
\end{tabular}

${ }^{a}$ Excluding two outliers.

is the source of the disagreement in the wavelength range 168$210 \mathrm{~nm}$ since the FP model exhibits less limb darkening than the QS model and the quiet disk which are in essential agreement. Furthermore, since the contrast $C_{\lambda}(1) \gtrsim 1$ in this wavelength range, the FP model is in error at a high level of significance.

In the wavelength ranges $142-168 \mathrm{~nm}$ and $210-265 \mathrm{~nm}$, the filter parameters for both models and the quiet disk agree, with one exception. As expected in the wavelength range 210 $265 \mathrm{~nm}$, Bonnet (1968) indicates stronger limb darkening than does Kjeldseth Moe \& Milone (1978) but the latter result agrees with the QS model, contrary to the conclusion of Greve \& Neckel (1996) noted in Sect. 4.4.

\section{Conclusions}

The complementary results of WWB and this paper confirm the advantages of having two different ultraviolet spectrometers on the Upper Atmosphere Research Satellite, SOLSTICE and SUSIM, and suggest that such multiplicity continues to be desirable for the near future. Together, for the first time, they describe the center-to-limb behavior of solar active regions at most far- and mid-ultraviolet wavelengths.

Because of systematic uncertainties the SOLSTICE results are limited to the wavelength range $120-170 \mathrm{~nm}$, while the SUSIM results are limited by sensitivity to the wavelength range $142-265 \mathrm{~nm}$. For the wavelengths common to both studies, the results are in good agreement given the lower resolution of the SUSIM and the resulting blending of continuum and emission lines in its observations. Where they overlap, both studies indicate that the center-to-limb variations of active regions and the quiet disk are similar. Misled by the models, WWB suggests that this agreement applies throughout the far ultraviolet (100-200 nm); our results show that it does not. Over the wavelength range $175-210 \mathrm{~nm}$, active regions exhibit much stronger limb darkening than does the quiet disk. At longer wavelengths in the mid ultraviolet $(210-265 \mathrm{~nm})$, active regions exhibit weak limb darkening, less than does the quiet disk.

The analyses in the two studies are complementary. The agreement between them will, we hope, engage the reader's interest in our less conventional approach. The interrelationships between the average intensities and center-to-limb variations in the multi-component model of WWB are complicated, requiring a non-linear-least-squares algorithm to solve; furthermore, the specific mathematical form chosen to describe the center-to-limb variations may, as the authors note, be inappropriate. On the other hand, our Fourier analysis addresses only the center-to-limb variations of the active regions but does so in terms of the model-independent filter parameter. Accordingly, these results could be used to constrain and simplify the model of WWB and improve their results.

We have also been able to compare our observational results with the model-atmosphere calculations for faint plage and quiet sun. We find excellent agreement at short and long wavelengths, but systematic disagreement in the wavelength range 168-210 nm, where our analysis shows stronger limb darkening than does the faint-plage model.

Our results suggest four directions for future research: (1) use of an area index like the Ca II K plage area in the Fourier analysis may allow determination of surface brightnesses for active regions; (2) application of the multi-component modeling of WWB to the SUSIM ultraviolet irradiances to take advantage of their greater wavelength coverage, using the results from the Fourier analysis to constrain and improve the modeling; (3) application of our Fourier analysis to the SOLSTICE ultraviolet irradiances can constrain and improve the existing modeling; (4) continued development of models, especially in the wavelength range 168-210, for comparison with observations.

Finally, our results combined with those of Donnelly \& Puga (1990) and DeLand \& Cebula (1998) illustrate an absence of accurate and convenient proxies for the solar ultraviolet irradiances, especially for the wavelength range $168-210 \mathrm{~nm}$. With the possible exception of the Mg I $285 \mathrm{~nm}$ feature, whose noise level in the SUSIM data is too large to be used in this analysis, no potential proxy exhibits the same degree of limb darkening, and has a time series showing the same level of fine detail.

The definitive description of solar ultraviolet irradiance variations awaits long-term imaging at the ultraviolet wavelengths considered above, as demonstrated by the SUMER imaging results for EUV and FUV wavelengths (Wilhelm et al. 1998; Dammasch et al. 1999). Ideally, solar UV imagers aboard each of two satellites in the same solar orbit separated by tens of degrees would provide the base data for a more accurate and complete description of the center-to-limb variation for various solar surface features. At present, time series of irradiances such as are provided by the SUSIM, SOLSTICE, and other similar past and future irradiance missions will remain the only observations available to help us understand the behaviors of active regions and the quiet disk at ultraviolet wavelengths. That understanding only can be attained by applying 
the widest variety of analytical methods to the available data. In this paper we have added an updated and refined Fourier analysis to our toolbox.

Acknowledgements. This work was supported by NASA-Defense Purchase Requests S14798D and S10108X. The authors wish to thank J. S. Morrill, K. Muglach, and the referee, W. Curdt, for their constructive comments.

\section{References}

Avrett, E. H., Machado, M. E., \& Kurucz, R. L. 1986, in The Lower Atmosphere of Solar Flares, ed. D. F. Neidig, National Solar Observatory, Sunspot NM, 216

Basri, G. S., Linsky, J. L., Bartoe, J.-D. F., Brueckner, G., \& VanHoosier, M. E. 1979, ApJ, 230, 924

Bonnet, R. 1968, Ann. Astrophys., 31, 597

Bracewell, R. 1965, The Fourier Transform and Its Applications (New York: McGraw-Hill)

Brekke, P., \& Kjeldseth-Moe, O. 1994a, ApJ, 431, L55

Brekke, P., \& Kjeldseth-Moe, O. 1994b, Sol. Phys., 150, 19

Brueckner, G. E., Edlow, K. L., Floyd, L. E., Lean, J. L., \& VanHoosier, M. E. 1993, J. Geophys. Res., 98, 10695

Chapman, G. A., \& McGuire, T. E. 1977, ApJ, 217, 657

Cook, J. W., \& Kjeldseth Moe, O. 1982, Sol. Phys., 76, 109

Cook, J. W., Brueckner, G. E., \& VanHoosier, M. E. 1980, J. Geophys. Res., 85, 2257

Coulman, C. E. 1985, ARA\&A, 23, 19

Crane, P. C. 1998, Sol. Phys., 177, 243

Crane, P. C. 2001, Sol. Phys., 203, 381

Dammasch, I. E., Wilhelm, K., Curdt, W., \& Schüle, U. 1999, in Proc. 9th European Meeting on Sol. Phys., Magnetic Fields and Solar Processes, ed. A. Wilson, ESA SP-448, 1165

DeLand, M. T., \& Cebula, R. P. 1998, Sol. Phys., 177, 79

Donnelly, R. F., \& Puga, L. C. 1990, Sol. Phys., 130, 369

Donnelly, R. F., Heath, D. F., \& Lean, J. L. 1982, J. Geophys. Res., 87,10318

Donnelly, R. F., Puga, L. C., Repoff, T. P., \& Lean, J. L. 1984, NOAA Tech. Memo. ERL ARL-126, NOAA ERL, Boulder, Colorado

Donnelly, R. F., Hinteregger, H. E., \& Heath, D. F. 1986, J. Geophys. Res., 91, 5567

Fligge, M., Solanki, S. K., \& Unruh, Y. C. 2000, Space Sci. Rev., 94, 139

Floyd, L., Brueckner, G., Crane, P., Prinz, P., \& Herring, L. 1997, in Proc. 31st ESLAB Symp., Correlated Phenomena at the Sun, in the Heliosphere and in Geospace, ed. A. Wilson, \& B. Fleck, ESTEC, 235

Floyd, L. E., Herring, L. C., Prinz, D. K., \& Crane, P. C. 1998a, Proc. SPIE, 3427, 445

Floyd, L. E., Reiser, P. A., Crane, P. C., et al. 1998b, Sol. Phys., 177, 79

Fontenla, J., White, O. R., Fox, P. A., Avrett, E. H., \& Kurucz, R. L. 1999, ApJ, 518, 480
Foukal, P., Harvey, K., \& Hill, F. 1991, ApJ, 383, L89

Gaizauskas, V., Harvey, K. L., Harvey, J. W., \& Zwaan, C. 1983, ApJ, 265,1056

Greve, A., \& Neckel, H. 1996, A\&AS, 120, 35

Harvey, J. W. 1984, in Solar Irradiance Variations on Active Region Time Scales, ed. B. J. LaBonte, G. A. Chapman, H. S. Hudson, \& R. C. Willson, NASA Conf. Publ., 2310, 197

Hersé, M. 1979, Sol. Phys., 63, 35

Hestroffer, D. 1997, A\&A, 327, 199

Hestroffer, D., \& Magnan, C. 1998, A\&A, 333, 338

Kiepenheuer, K. O. 1953, in The Sun, ed. G. P. Kuiper (Chicago: University of Chicago), 338

Kjeldseth Moe, O., \& Milone, E. F. 1978, ApJ, 226, 301

Lean, J. L. 1984, J. Geophys. Res., 89, 1

Lean, J. 1987, J. Geophys. Res., 92, 839

Lean, J. L. 1988, Adv. Space Res., 8, 85

Lean, J. L., \& Skumanich, A. 1983, J. Geophys. Res., 88, 5751

Lean, J. L., White, O. R., Livingston, W. C., et al. 1982, J. Geophys. Res., 87, 10307

Lean, J., VanHoosier, M., Brueckner, G., et al. 1992, Geophys. Res. Lett., 19, 2203

Maunder, E. W. 1922, MNRAS, 82, 534

Morrill, J. S., Dere, K. P., \& Korendyke, C. M. 2001, ApJ, 557, 854

Neckel, H., \& Labs, D. 1994, Sol. Phys., 153, 91

Petro, L. D., Foukal, P. V., Rosen, W. A., Kurucz, R. L., \& Pierce, A. K. 1984, ApJ, 283, 426

Prinz, D. K. 1974, ApJ, 187, 369

Roberts, D. H., \& Lehár, J. 1994, Priv. Comm.

Roberts, D. H., Lehár, J., \& Dreher, J. W. 1987, AJ, 93, 968

Rottman, G. J., Woods, T. N., \& Sparn, T. P. 1993, J. Geophys. Res., 98, 10667

Roussel-Dupré, D. 1982, ApJ, 256, 284

Samain, D. 1979, A\&A, 74, 225

Samain, D. 1980, ApJS, 44, 273

Shao, M., \& Colavita, M. M. 1992, ARA\&A, 30, 457

Shapiro, R. 1965a, J. Geophys. Res., 70, 245

Shapiro, R. 1965b, J. Geophys. Res., 70, 3581

Skumanich, A., Lean, J. L., White, O. R., \& Livingston, W. C. 1984, ApJ, 282, 776

Thompson, A. R., Moran, J. M., \& Swenson, G. W. 1986, Interferometry and Synthesis in Radio Astronomy (New York: Wiley)

Unruh, Y. C., Solanki, S. K., \& Fligge, M. 1999, A\&A, 345, 635

Wang, H., \& Zirin, H. 1987, Sol. Phys., 110, 281

Ward, F., \& Shapiro, R. 1961, Ann. New York Acad. Sci., 95, 200

Ward, F., \& Shapiro, R. 1962, J. Geophys. Res., 67, 541

Wilhelm, K., Lemaire, P., Dammasch, I. E., et al. 1998, A\&A, 334, 685

Woods, T. N., Rottman, G. J., \& Ucker, G. 1993, J. Geophys. Res., 98 , 10679

Worden, J. R., White, O. R., \& Woods, T. N. 1998, ApJ, 496, 998

Worden, J. R., Woods, T. N., \& Bowman, K. W. 2001, ApJ, 560, 1020 (WWB) 